\title{
THE HOPF LEMMA FOR THE SCHRÖDINGER OPERATOR
}

\author{
AUGUSTO C. PONCE AND NICOLAS WILMET
}

\begin{abstract}
We prove the Hopf boundary point lemma for solutions of the Dirichlet problem involving the Schrödinger operator $-\Delta+V$ with a nonnegative potential $V$ which merely belongs to $L_{\text {loc }}^{1}(\Omega)$. More precisely, if $u \in W_{0}^{1,2}(\Omega) \cap L^{2}(\Omega ; V \mathrm{~d} x)$ satisfies $-\Delta u+V u=f$ on $\Omega$ for some nonnegative datum $f \in L^{\infty}(\Omega), f \not \equiv 0$, then we show that at every point $a \in \partial \Omega$ where the classical normal derivative $\partial u(a) / \partial n$ exists and satisfies the Poisson representation formula, one has $\partial u(a) / \partial n>0$ if and only if the boundary value problem

$$
\left\{\begin{array}{rlrl}
-\Delta v+V v=0 & & \text { in } \Omega, \\
v & =\nu & & \text { on } \partial \Omega,
\end{array}\right.
$$

involving the Dirac measure $\nu=\delta_{a}$ has a solution. More generally, we characterize the nonnegative finite Borel measures $\nu$ on $\partial \Omega$ for which the boundary value problem above has a solution in terms of the set where the Hopf lemma fails.
\end{abstract}

\section{InTRODUCTION AND MAIN RESUlts}

Let $\Omega$ be a bounded connected open subset of $\mathbb{R}^{N}$ with smooth boundary and let $V \in L^{\infty}(\Omega)$ be a nonnegative function. The weak maximum principle ensures that the distributional solution $u \in C^{1}(\bar{\Omega})$ of the Dirichlet problem

$$
\left\{\begin{aligned}
-\Delta u+V u=f & \text { in } \Omega, \\
u=0 & \text { on } \partial \Omega,
\end{aligned}\right.
$$

satisfies $u \geq 0$ on $\Omega$ whenever $f \in L^{\infty}(\Omega)$ is a nonnegative function; see Lemma 2.2 below. From the minimality of $u$ on $\partial \Omega$, the normal derivative of $u$ with respect to the inward unit normal vector $n$ thus verifies $\partial u / \partial n \geq 0$ on $\partial \Omega$. When $f \not \equiv 0$, the classical Hopf lemma (see [10, Lemma 6.4.2] or [11, Lemma 3.4]) gives the stronger conclusion

$$
\frac{\partial u}{\partial n}>0 \text { on } \partial \Omega \text {. }
$$

Boundedness of $V$ is an important element to obtain (1.2) as it allows one to construct a positive minorant of $u$ on $\Omega$ with positive normal derivative

2010 Mathematics Subject Classification. Primary: 35J10, 35D05, 35G15; Secondary: $35 \mathrm{C} 15$.

Key words and phrases. Schrödinger operator, Hopf lemma, boundary value problem, singular potential, measure datum. 
at any given point on $\partial \Omega$. To understand in what respect this assumption on $V$ can be relaxed, we assume henceforth that

$$
V \in L_{\text {loc }}^{1}(\Omega) \text { and } V \geq 0 \text { almost everywhere on } \Omega,
$$

but we restrict ourselves to the class of nonnegative data $f \in L^{\infty}(\Omega)$. In this setting, a solution of (1.1) is a function $u$ that belongs to $W_{0}^{1,2}(\Omega) \cap$ $L^{2}(\Omega ; V \mathrm{~d} x)$ and satisfies the equation

$-\Delta u+V u=f \quad$ in the sense of distributions in $\Omega$.

Observe that $u$ is the unique minimizer of the energy functional

$$
E(z)=\frac{1}{2} \int_{\Omega}\left(|\nabla z|^{2}+V z^{2}\right) \mathrm{d} x-\int_{\Omega} f z \mathrm{~d} x
$$

with $z \in W_{0}^{1,2}(\Omega) \cap L^{2}(\Omega ; V \mathrm{~d} x)$.

As the solution of (1.1) need not be $C^{1}$, nor even continuous, due to some possible singularity from $V$, we first need to address the pointwise meaning of the normal derivative $\partial u / \partial n$. Since $u$ is the difference between a continuous and a bounded superharmonic function, every $x \in \Omega$ is a Lebesgue point and the precise representative of $u$ satisfies the following representation formula in terms of the Green function $G$ of $-\Delta$ on $\Omega$ :

$$
\hat{u}(x)=\int_{\Omega} G(x, y)(-\Delta u(y)) \mathrm{d} y \quad \text { for every } x \in \Omega .
$$

Then, from a formal computation, one presumably gets at a point $a \in \partial \Omega$ :

$$
\frac{\partial \hat{u}}{\partial n}(a)=\int_{\Omega} K(a, y)(-\Delta u(y)) \mathrm{d} y,
$$

where $K:=\partial G / \partial n$ denotes the Poisson kernel of $-\Delta$ on $\Omega$. This formula can be rigorously justified when $V \in L^{\infty}(\Omega)$, and then $\Delta u \in L^{\infty}(\Omega)$, using standard estimates on $G$.

There is no reason why (1.3) should remain valid in general as we do not assume any particular behaviour of $V$ near $\partial \Omega$. We show nevertheless that, for any fixed $V$, there is a common property which is shared by all nontrivial solutions of (1.1) with nonnegative $f \in L^{\infty}(\Omega)$. To this end, let $\zeta_{1}$ be the solution of (1.1) with constant density $f \equiv 1$ and define the set

$$
\mathcal{N}=\left\{\begin{array}{l|l}
a \in \partial \Omega & \begin{array}{l}
\text { the classical normal derivative } \partial \widehat{\zeta_{1}} / \partial n \text { exists at } a \\
\text { and }(1.3) \text { is valid with } u=\zeta_{1}
\end{array}
\end{array}\right\} .
$$

To simplify the notation, we do not explicit the dependence of $\mathcal{N}$ on $V$.

We prove

Theorem 1. For every nonnegative function $f \in L^{\infty}(\Omega), f \not \equiv 0$, the solution $u$ of (1.1) involving $f$ has a classical normal derivative at $a \in \partial \Omega$ that satisfies (1.3) if and only if $a \in \mathcal{N}$. 
The set $\mathcal{N}$ thus provides one with a common ground where a normal derivative exists, independently of the solution of (1.1). We can now address the question of whether the Hopf lemma is valid on $\mathcal{N}$. We rely on the characterization of the set of points $a \in \partial \Omega$ for which the boundary value problem

$$
\left\{\begin{array}{rlrl}
-\Delta v+V v=0 & & \text { in } \Omega, \\
v & =\delta_{a} & & \text { on } \partial \Omega,
\end{array}\right.
$$

involving the Dirac measure $\delta_{a}$ has a distributional solution in the sense that $v \in L^{1}(\Omega)$ is such that $V v \in L^{1}\left(\Omega ; d_{\partial \Omega} \mathrm{d} x\right)$ and satisfies

$$
\frac{\partial \zeta}{\partial n}(a)=\int_{\Omega} v(-\Delta \zeta+V \zeta) \mathrm{d} x
$$

for every $\zeta \in C^{\infty}(\bar{\Omega})$ with $\zeta=0$ on $\partial \Omega$, where $d_{\partial \Omega}: \Omega \rightarrow \mathbb{R}_{+}$is the distance to the boundary. When the test function $\zeta$ is non-identically zero and satisfies $-\Delta \zeta+V \zeta \geq 0$ on $\Omega$, it follows from (1.5) and the strong maximum principle for the Schrödinger operator with potential in $L_{\text {loc }}^{1}$ (see [1, Théorème 9], [5, Theorem 1] or [19]) that $\partial \zeta(a) / \partial n>0$. It is therefore reasonable to expect the validity of the Hopf lemma for (1.1) on the set of points $a \in \partial \Omega$ for which the boundary value problem (1.4) has a solution. This motivates the following

Definition 1.1. The exceptional boundary set $\Sigma$ associated to $-\Delta+V$ is the set of points $a \in \partial \Omega$ for which the boundary value problem (1.4) with datum $\delta_{a}$ does not have a distributional solution.

We can now state the Hopf lemma on $\mathcal{N}$ :

Theorem 2. Let $u$ be the solution of (1.1) for some nonnegative datum $f \in L^{\infty}(\Omega), f \not \equiv 0$. For every $a \in \mathcal{N}$, we have

$$
\frac{\partial \hat{u}}{\partial n}(a)>0 \quad \text { if and only if } a \notin \Sigma .
$$

In the case where $V \in L^{q}(\Omega)$ for some $q>N$, one has $\mathcal{N}=\partial \Omega$ and $\Sigma=\emptyset$. Hence,

$$
\frac{\partial \hat{u}}{\partial n}(a)>0 \quad \text { for every } a \in \partial \Omega ;
$$

see Corollary 7.2 below. As one can expect, the validity of the Hopf lemma depends on the behaviour of $V$ near the boundary. For example, under the assumption that

$$
V \leq C / d_{\partial \Omega}^{2} \quad \text { almost everywhere on } \Omega
$$

for some constant $C \geq 0$, Ancona established in [2] (see also the Appendix in [20]) a beautiful characterization of the set of points where (1.4) has a solution: $a \in \partial \Omega \backslash \Sigma$ if and only if the Poisson kernel of $-\Delta$ at $a$ is a supersolution of (1.1). Using his result and the pointwise behaviour of $K$, we can state the following 
Corollary 1.1. Assume that $V$ satisfies (1.6) and let $u$ be the solution of (1.1) for some nonnegative datum $f \in L^{\infty}(\Omega), f \not \equiv 0$. For every $a \in \mathcal{N}$, we have

$$
\frac{\partial \hat{u}}{\partial n}(a)>0 \quad \text { if and only if } \quad \int_{\Omega} \frac{d_{\partial \Omega}^{2}(y)}{|y-a|^{N}} V(y) \mathrm{d} y<+\infty .
$$

Quadratic blow-up of the potential as in (1.6) is a threshold for the validity of the Hopf lemma. More precisely,

Corollary 1.2. Assume that $V$ satisfies

$$
V \geq C^{\prime} / d_{\partial \Omega}^{2} \quad \text { almost everywhere on } \Omega
$$

for some $C^{\prime}>0$. Then $\mathcal{N}=\partial \Omega$ and, for every solution $u$ of (1.1) with $f \in L^{\infty}(\Omega)$, we have

$$
\frac{\partial \hat{u}}{\partial n}=0 \quad \text { on } \partial \Omega .
$$

Corollary 1.2 is a consequence of our Theorem 1 and a result from Díaz [8] which establishes the existence of a bounded nonnegative eigenfunction $u$ for $-\Delta+V$ that satisfies a Dirichlet problem of the type (1.1) and such that $\partial \hat{u}(a) / \partial n=0$ for every $a \in \partial \Omega$.

Although we have introduced the exceptional set $\Sigma$ by dealing with Dirac masses on $\partial \Omega$, the set $\Sigma$ allows one to characterize all nonnegative finite Borel measures $\nu$ on $\partial \Omega$ for which the boundary value problem

$$
\left\{\begin{aligned}
-\Delta v+V v & =0 & & \text { in } \Omega, \\
v & =\nu & & \text { on } \partial \Omega,
\end{aligned}\right.
$$

has a distributional solution. This is the content of our next theorem that extends a previous result by Véron and Yarur [20]:

Theorem 3. The boundary value problem (1.7) associated to a nonnegative finite Borel measure $\nu$ on $\partial \Omega$ has a distributional solution if and only if $\nu(\Sigma)=0$.

The proof of Theorem 3 is inspired by the recent paper of Orsina and the first author [17] concerning the failure of the strong maximum principle for the Schrödinger operator $-\Delta+V$ in the case where $V$ is merely a nonnegative Borel measurable function. In this respect, we introduce in Section 2 a notion of pointwise normal derivative for solutions of (1.1) that is defined everywhere on $\partial \Omega$, but possibly depends on the potential $V$. In Section 3, we present a counterpart for (1.7) of the notion of duality solution introduced by Malusa and Orsina [13]. The exceptional boundary set $\Sigma$ is then identified in Section 4 with the set of boundary points at which all such normal derivatives vanish. Using the tools developed in Sections 3 and 4, we prove Theorem 3 in Section 5. Theorems 1 and 2 are proved in Sections 6 and 7, respectively. 


\section{Pointwise normal derivative associated to the Schrödinger OPERATOR}

A property that is common to all solutions of (1.1) concerns the existence of a distributional normal derivative as an element in $L^{1}(\partial \Omega)$. This is a general feature that relies on the facts that

$$
u \in W_{0}^{1,1}(\Omega) \text { and } \Delta u \text { is a finite Borel measure on } \Omega .
$$

Brezis and the first author proved in [7] that in this general setting there exists a function in $L^{1}(\partial \Omega)$, which is denoted by $\partial u / \partial n$ and coincides with the classical normal derivative when $u$ is a $C^{2}$ function, that satisfies

$$
\left\|\frac{\partial u}{\partial n}\right\|_{L^{1}(\partial \Omega)} \leq|\Delta u|(\Omega)
$$

and

$$
\int_{\Omega} \nabla u \cdot \nabla \psi \mathrm{d} x=-\int_{\Omega} \psi \Delta u-\int_{\partial \Omega} \frac{\partial u}{\partial n} \psi \mathrm{d} \sigma \quad \text { for every } \psi \in C^{\infty}(\bar{\Omega}),
$$

where $\sigma=\mathcal{H}^{N-1}\lfloor\partial \Omega$ is the surface measure on $\partial \Omega$; see [7, Theorem 1.2] or [18, Proposition 7.3]. We recall that $n$ is the inward unit normal vector, which explains the minus sign in front of the second integral in the righthand side of (2.1). When $u \geq 0$ almost everywhere on $\Omega$, one additionally has

$$
\frac{\partial u}{\partial n} \geq 0 \quad \text { almost everywhere on } \partial \Omega ;
$$

see [7, Corollary 6.1] or [18, Lemma 12.15]. Since the mapping

$$
\left\{u \in W_{0}^{1,1}(\Omega): \Delta u \in L^{1}(\Omega)\right\} \rightarrow L^{1}(\partial \Omega): u \mapsto \frac{\partial u}{\partial n}
$$

is linear, such a property yields a handy comparison principle: if $v$ and $w$ both satisfy (1.1), with possibly different potentials $V$ and data $f$, and if $v \leq w$ almost everywhere on $\Omega$, then

$$
\frac{\partial v}{\partial n} \leq \frac{\partial w}{\partial n} \quad \text { almost everywhere on } \partial \Omega .
$$

More specific to solutions of (1.1), we show that there is a notion of pointwise normal derivative that is adapted to the Schrödinger operator $-\Delta+V$ and used in the proofs of Theorems 1 to 3 . For this purpose, let $\left(V_{k}\right)$ be a nondecreasing sequence of nonnegative functions in $L^{\infty}(\Omega)$ that converges almost everywhere to $V$ on $\Omega$. The construction of this pointwise normal derivative relies on the main result of this section which is

Proposition 2.1. Let $u$ be the solution of (1.1) associated to $f \in L^{\infty}(\Omega)$ and denote by $u_{k}$ the solution of

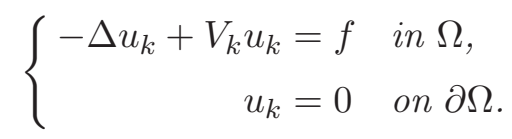

Then 
(i) $u_{k} \rightarrow u$ and $V_{k} u_{k} \rightarrow V u$ in $L^{1}(\Omega)$ and almost everywhere on $\Omega$;

(ii) $\left(\partial u_{k} / \partial n\right)$ is uniformly bounded on $\partial \Omega$;

(iii) $\left(\partial u_{k} / \partial n\right)$ converges pointwise to a function $g: \partial \Omega \rightarrow \mathbb{R}$ such that $g=\partial u / \partial n$ almost everywhere on $\partial \Omega$ and, for every $N<p \leq \infty$,

$$
\|g\|_{L^{\infty}(\partial \Omega)} \leq C\|f\|_{L^{p}(\Omega)}
$$

with a constant $C>0$ depending on $p$ and $\Omega$. Moreover, $g \geq 0$ on $\partial \Omega$ whenever $f \geq 0$ almost everywhere on $\Omega$.

Since $V_{k}$ is bounded, we have $u_{k} \in C^{1}(\bar{\Omega})$ and in particular the classical normal derivative $\partial u_{k} / \partial n$ is well-defined on $\partial \Omega$. To see why this is true, let $w \in C^{\infty}(\bar{\Omega})$ be the solution of

$$
\left\{\begin{aligned}
-\Delta w & =|f| & & \text { in } \Omega, \\
w & =0 & & \text { on } \partial \Omega .
\end{aligned}\right.
$$

The weak maximum principle implies that $\left|u_{k}\right| \leq w$ almost everywhere on $\Omega$; thus $u_{k} \in L^{\infty}(\Omega)$. Since $V_{k}$ and $f$ are bounded, we have $\Delta u_{k} \in L^{\infty}(\Omega)$, hence $u_{k} \in W^{2, p}(\Omega)$ for every $1<p<\infty$; see [11, Theorem 9.15 and Lemma 9.17]. Taking any $p>N$, it follows from the Morrey-Sobolev embedding theorem that $u_{k} \in C^{1}(\bar{\Omega})$; see $[21$, Theorem 6.4.4]. In addition, one has the estimate

$$
\|w\|_{C^{1}(\bar{\Omega})} \leq C\|\Delta w\|_{L^{p}(\Omega)}=C\|f\|_{L^{p}(\Omega)}
$$

for some constant $C>0$ depending on $p$ and $\Omega$. Since

$$
\left|\frac{\partial u_{k}}{\partial n}\right| \leq \frac{\partial w}{\partial n} \quad \text { on } \partial \Omega
$$

one deduces from (2.4) that

$$
\left\|\frac{\partial u_{k}}{\partial n}\right\|_{L^{\infty}(\Omega)} \leq\|w\|_{C^{1}(\bar{\Omega})} \leq C\|f\|_{L^{p}(\Omega)} .
$$

Using Proposition 2.1, we then define the pointwise normal derivative of $u$ with respect to $-\Delta+V$ as

$$
\widehat{\partial u}(a):=g(a) \quad \text { for every } a \in \partial \Omega \text {. }
$$

At first sight, this definition could depend on the choice of approximation $\left(V_{k}\right)$ like

$$
V_{k}=\min \{V, k\},
$$

but as we shall see later on it does not; see Remark 5.1. As a consequence of assertion (iii) in Proposition 2.1, $\widehat{\partial u} / \partial n$ is a distributional normal derivative of $u$.

Before proceeding with the proof of Proposition 2.1, we recall standard estimates for solutions of the Dirichlet problem

$$
\left\{\begin{aligned}
-\Delta u+V u=\mu & \text { in } \Omega, \\
u=0 & \text { on } \partial \Omega,
\end{aligned}\right.
$$


where $\mu \in L^{1}(\Omega)$. By a solution of (2.6), we mean a function $u \in W_{0}^{1,1}(\Omega) \cap$ $L^{1}(\Omega ; V \mathrm{~d} x)$ that satisfies the equation in the sense of distributions in $\Omega$. For all $1 \leq p<\frac{N}{N-1}$, the solution exists, is unique and belongs to $W_{0}^{1, p}(\Omega)$ with

$$
\|u\|_{W^{1, p}(\Omega)} \leq C\|\mu\|_{L^{1}(\Omega)}
$$

for some constant $C>0$ depending on $p$ and $\Omega$. This can be deduced from elliptic estimates due to Littman, Stampacchia and Weinberger [12, Theorem 5.1] and from the absorption estimate

$$
\|V u\|_{L^{1}(\Omega)} \leq\|\mu\|_{L^{1}(\Omega)} .
$$

The latter inequality can be obtained using as test function a suitable approximation of the sign function $\operatorname{sgn} u$; see [4, Proposition 4.B.3] or [18, Proposition 21.5].

The weak maximum principle for (2.6) that is mentioned in the introduction is justified by the following

Lemma 2.2. Let $u$ be the solution of (2.6) involving $\mu \in L^{1}(\Omega)$. If $\mu \geq 0$ almost everywhere on $\Omega$, then $u \geq 0$ almost everywhere on $\Omega$.

The proof of Lemma 2.2 relies on a variant of Kato's inequality: if $w \in$ $L^{1}(\Omega), h \in L^{1}\left(\Omega, d_{\partial \Omega} \mathrm{d} x\right)$ and $\nu \in \mathcal{M}(\partial \Omega)$ satisfy

$$
-\int_{\Omega} w \Delta \zeta \mathrm{d} x=\int_{\Omega} h \zeta \mathrm{d} x+\int_{\partial \Omega} \frac{\partial \zeta}{\partial n} \mathrm{~d} \nu \quad \text { for every } \zeta \in C_{0}^{\infty}(\bar{\Omega}),
$$

then

$-\int_{\Omega} w^{+} \Delta \zeta \mathrm{d} x \leq \int_{\{w \geq 0\}} h \zeta \mathrm{d} x+\int_{\partial \Omega} \frac{\partial \zeta}{\partial n} \mathrm{~d} \nu^{+} \quad$ for every $\zeta \in C_{0}^{\infty}(\bar{\Omega}), \zeta \geq 0$ on $\bar{\Omega}$

see [14, Lemma 1.5] or [15, Proposition 1.5.9]. Here $\mathcal{M}(\partial \Omega)$ denotes the vector space of finite Borel measures on $\partial \Omega$ and

$$
C_{0}^{\infty}(\bar{\Omega})=\left\{\zeta \in C^{\infty}(\bar{\Omega}): \zeta=0 \text { on } \partial \Omega\right\} .
$$

When $\nu=0$, the integral identity (2.9) implicitly encodes the fact that $w=0$ on $\partial \Omega$ in an average sense as test functions need not have compact support in $\Omega$; see [18, Proposition 20.2] and also [9] for related questions. To deduce the weak maximum principle it now suffices to take $w=-u, h=V u-\mu$ and $\nu=0$, and then (2.10) becomes

$$
-\int_{\Omega}(-u)^{+} \Delta \zeta \mathrm{d} x \leq 0 \quad \text { for every } \zeta \in C_{0}^{\infty}(\bar{\Omega}), \zeta \geq 0 \text { on } \bar{\Omega} .
$$

One last ingredient involved in the proof of Proposition 2.1 is the following comparison principle: 
Lemma 2.3. Let $V_{1}, V_{2} \in L_{\mathrm{loc}}^{1}(\Omega)$ be two nonnegative functions such that $V_{1} \leq V_{2}$ almost everywhere on $\Omega$, and let $u_{i} \in L^{1}(\Omega) \cap L^{1}\left(\Omega ; V_{i} d_{\partial \Omega} \mathrm{d} x\right)$, with $i \in\{1,2\}$, be two nonnegative functions such that

$$
-\int_{\Omega}\left(u_{2}-u_{1}\right) \Delta \zeta \mathrm{d} x+\int_{\Omega}\left(V_{2} u_{2}-V_{1} u_{1}\right) \zeta \mathrm{d} x=0 \quad \text { for every } \zeta \in C_{0}^{\infty}(\bar{\Omega}) .
$$

Then $u_{2} \leq u_{1}$ almost everywhere on $\Omega$.

Lemma 2.3 can be deduced using Kato's inequality as above by taking $w=u_{2}-u_{1}$.

Proof of Proposition 2.1. We assume that $f$ is nonnegative; the general case follows by solving the Dirichlet problem with the positive and negative parts of $f$, and then conclude using the linearity of the equation in (1.1) and uniqueness of solutions. Hence, by the weak maximum principle, $u$ and $u_{k}$ are nonnegative. Since $u$ satisfies

$$
-\Delta u+V_{k} u=f-\left(V-V_{k}\right) u \text { in the sense of distributions in } \Omega,
$$

we have

$-\Delta\left(u_{k}-u\right)+V_{k}\left(u_{k}-u\right)=\left(V-V_{k}\right) u$ in the sense of distributions in $\Omega$.

One deduces from (2.7) applied to $u_{k}-u$ that

$$
\left\|u_{k}-u\right\|_{L^{1}(\Omega)} \leq C\left\|\left(V-V_{k}\right) u\right\|_{L^{1}(\Omega)} .
$$

By Lebesgue's dominated convergence theorem, the right-hand side of this inequality tends to 0 as $k \rightarrow \infty$. Hence $u_{k} \rightarrow u$ in $L^{1}(\Omega)$. Since $\left(u_{k}\right)$ is non-increasing as a consequence of Lemma 2.3, the convergence also holds everywhere on $\Omega$. The triangle inequality and the absorption estimate (2.8) applied to $u_{k}-u$ imply that

$\left\|V_{k} u_{k}-V u\right\|_{L^{1}(\Omega)} \leq\left\|V_{k}\left(u_{k}-u\right)\right\|_{L^{1}(\Omega)}+\left\|\left(V_{k}-V\right) u\right\|_{L^{1}(\Omega)} \leq 2\left\|\left(V-V_{k}\right) u\right\|_{L^{1}(\Omega)}$, and then $V_{k} u_{k} \rightarrow V u$ in $L^{1}(\Omega)$.

By comparison of normal derivatives, the sequence $\left(\partial u_{k} / \partial n\right)$ is nonincreasing and nonnegative. In particular, it is uniformly bounded on $\partial \Omega$ and converges in $L^{1}(\partial \Omega)$ and everywhere on $\partial \Omega$ to some nonnegative bounded measurable function $g: \partial \Omega \rightarrow \mathbb{R}$. Let us show that

$$
g=\frac{\partial u}{\partial n} \text { almost everywhere on } \partial \Omega,
$$

where $\partial u / \partial n$ is the distributional normal derivative of $u$. For this purpose, we recall that each $u_{k}$ satisfies

$$
\int_{\Omega} \nabla u_{k} \cdot \nabla \psi \mathrm{d} x=\int_{\Omega} f \psi \mathrm{d} x-\int_{\Omega} V_{k} u_{k} \psi \mathrm{d} x-\int_{\partial \Omega} \frac{\partial u_{k}}{\partial n} \psi \mathrm{d} \sigma
$$

for every $\psi \in C^{\infty}(\bar{\Omega})$. By standard interpolation, which in this case follows from an integration by parts, one also has the estimate

$$
\left\|\nabla u_{k}\right\|_{L^{2}(\Omega)}^{2} \leq\left\|u_{k}\right\|_{L^{\infty}(\Omega)}\left\|\Delta u_{k}\right\|_{L^{1}(\Omega)} ;
$$


see [18, Lemma 5.8]. We claim that the right-hand side of this inequality is bounded. Indeed, as $\left(u_{k}\right)$ is non-increasing, it is bounded from above by $u_{0}$. On the other hand, we deduce from the triangle inequality and the absorption estimate (2.8) that

$$
\left\|\Delta u_{k}\right\|_{L^{1}(\Omega)} \leq\|f\|_{L^{1}(\Omega)}+\left\|V_{k} u_{k}\right\|_{L^{1}(\Omega)} \leq 2\|f\|_{L^{1}(\Omega)},
$$

which validates our claim.

Since $\left(\nabla u_{k}\right)$ is bounded in $L^{2}\left(\Omega ; \mathbb{R}^{N}\right)$ and $u_{k} \rightarrow u$ in $L^{1}(\Omega)$, we have

$$
\nabla u_{k} \rightarrow \nabla u \text { weakly in } L^{2}\left(\Omega ; \mathbb{R}^{N}\right) \text {. }
$$

Taking the limit as $k \rightarrow \infty$ in (2.11), we obtain

$$
\int_{\partial \Omega} \frac{\partial u}{\partial n} \psi \mathrm{d} \sigma=\int_{\partial \Omega} g \psi \mathrm{d} \sigma \quad \text { for every } \psi \in C^{\infty}(\bar{\Omega}) .
$$

Hence $\partial u / \partial n=g$ almost everywhere on $\partial \Omega$. The estimate

$$
\|g\|_{L^{\infty}(\partial \Omega)} \leq C\|f\|_{L^{p}(\Omega)}
$$

follows from (2.5) since $0 \leq g \leq \partial u_{k} / \partial n$ on $\partial \Omega$.

\section{Duality solution With Measure Data on the Boundary}

We investigate the boundary value problem (1.7) involving a finite Borel measure $\nu$ on $\partial \Omega$ by comparing two notions of solution based on different choices of test functions.

Definition 3.1. A function $v \in L^{1}(\Omega)$ is a distributional solution of (1.7) with datum $\nu \in \mathcal{M}(\partial \Omega)$ whenever $V v \in L^{1}\left(\Omega ; d_{\partial \Omega} \mathrm{d} x\right)$ and

$$
\int_{\Omega} v(-\Delta \zeta+V \zeta) \mathrm{d} x=\int_{\partial \Omega} \frac{\partial \zeta}{\partial n} \mathrm{~d} \nu \text { for every } \zeta \in C_{0}^{\infty}(\bar{\Omega}) .
$$

The boundary value problem for this type of solutions has been studied by Véron and Yarur [20] with nonnegative potentials $V \in L_{\text {loc }}^{\infty}(\Omega)$. In particular, the authors prove that nonnegative measures for which (1.7) has a solution cannot charge $\Sigma$; see [20, Theorem 4.4]. Their approach is based on the careful study of some capacity associated to the Poisson kernel of $-\Delta$ on $\Omega$. In our case, we rely instead on the concept of duality solution in the spirit of the work of Malusa and Orsina [13] that has its roots in the seminal paper of Littman, Stampacchia and Weinberger [12].

Definition 3.2. A function $v \in L^{1}(\Omega)$ is a duality solution of (1.7) with datum $\nu \in \mathcal{M}(\partial \Omega)$ whenever

$$
\int_{\Omega} v f \mathrm{~d} x=\int_{\partial \Omega} \frac{\widehat{\partial \zeta_{f}}}{\partial n} \mathrm{~d} \nu \text { for every } f \in L^{\infty}(\Omega)
$$

where $\zeta_{f}$ is the solution of (1.1) with datum $f$.

Existence of duality solutions is a straightforward consequence of the Riesz representation theorem: 
Proposition 3.1. The boundary value problem (1.7) has a unique duality solution for every datum $\nu \in \mathcal{M}(\partial \Omega)$.

Proof. Let $N<p<\infty$. It follows from Proposition 2.1 that, for every $f \in L^{\infty}(\Omega)$,

$$
\left|\int_{\partial \Omega} \frac{\widehat{\partial \zeta_{f}}}{\partial n} \mathrm{~d} \nu\right| \leq\|\nu\|_{\mathcal{M}(\partial \Omega)}\left\|\frac{\widehat{\partial \zeta_{f}}}{\partial n}\right\|_{L^{\infty}(\partial \Omega)} \leq C\|\nu\|_{\mathcal{M}(\partial \Omega)}\|f\|_{L^{p}(\Omega)}
$$

where

$$
\|\nu\|_{\mathcal{M}(\partial \Omega)}:=|\nu|(\partial \Omega) .
$$

Hence, the linear functional

$$
L^{\infty}(\Omega) \rightarrow \mathbb{R}: f \mapsto \int_{\partial \Omega} \frac{\widehat{\partial \zeta_{f}}}{\partial n} \mathrm{~d} \nu
$$

is continuous on $L^{\infty}(\Omega)$, endowed with the $L^{p}$ norm. The Riesz representation theorem implies the existence of a unique $v \in L^{p^{\prime}}(\Omega)$ such that

$$
\int_{\Omega} v f \mathrm{~d} x=\int_{\partial \Omega} \frac{\widehat{\partial \zeta_{f}}}{\partial n} \mathrm{~d} \nu \text { for every } f \in L^{\infty}(\Omega),
$$

where $p^{\prime}=\frac{p}{p-1}$ is the conjugate exponent with respect to $p$. Hence $v$ is the unique duality solution of (1.7) involving $\nu$.

We now prove that distributional solutions are duality solutions:

Proposition 3.2. If $v$ is a distributional solution of (1.7) with datum $\nu \in$ $\mathcal{M}(\partial \Omega)$, then $v$ is also a duality solution of (1.7) with datum $\nu$.

For the proof of Proposition 3.2, we need a couple of lemmas. We begin with

Lemma 3.3. Assume that (1.7) has a distributional solution $v$ with datum $\nu \in \mathcal{M}(\partial \Omega)$ and let $v_{k}$ be the distributional solution of

$$
\left\{\begin{aligned}
-\Delta v_{k}+V_{k} v_{k}=0 & \text { in } \Omega, \\
v_{k}=\nu & \text { on } \partial \Omega .
\end{aligned}\right.
$$

Then $v_{k} \rightarrow v$ in $L^{1}(\Omega)$.

Proof of Lemma 3.3. First notice that the function $v-v_{k}$ satisfies

$$
\int_{\Omega}\left(v-v_{k}\right)\left(-\Delta \zeta+V_{k} \zeta\right) \mathrm{d} x=\int_{\Omega}\left(V_{k}-V\right) v \zeta \mathrm{d} x \quad \text { for every } \zeta \in C_{0}^{\infty}(\bar{\Omega}) .
$$

Kato's inequality (2.10) applied to $v-v_{k}$ and $-\left(v-v_{k}\right)$ with $\nu=0$ implies that

$$
\int_{\Omega}\left|v-v_{k}\right|\left(-\Delta \zeta+V_{k} \zeta\right) \mathrm{d} x \leq \int_{\Omega} \operatorname{sgn}\left(v-v_{k}\right)\left(V_{k}-V\right) v \zeta \mathrm{d} x
$$

and then

$$
\int_{\Omega}\left|v-v_{k}\right|\left(-\Delta \zeta+V_{k} \zeta\right) \mathrm{d} x \leq \int_{\Omega} \zeta\left(V-V_{k}\right)|v| \mathrm{d} x
$$


for every $\zeta \in C_{0}^{\infty}(\bar{\Omega}), \zeta \geq 0$ on $\bar{\Omega}$. We take as test function the unique solution of

$$
\left\{\begin{aligned}
-\Delta \theta=1 & \text { in } \Omega, \\
\theta=0 & \text { on } \partial \Omega .
\end{aligned}\right.
$$

Observing that $V_{k} \theta \geq 0$ and $\theta \leq\|\nabla \theta\|_{L^{\infty}(\Omega)} d_{\partial \Omega}$ on $\Omega$, we obtain the estimate

$$
\left\|v_{k}-v\right\|_{L^{1}(\Omega)} \leq\|\nabla \theta\|_{L^{\infty}(\Omega)}\left\|\left(V-V_{k}\right) v\right\|_{L^{1}\left(\Omega ; d_{\partial \Omega} \mathrm{d} x\right)} .
$$

Since $0 \leq V_{k} \leq V$ and $V v \in L^{1}\left(\Omega ; d_{\partial \Omega} \mathrm{d} x\right)$, Lebesgue's dominated convergence theorem implies that

$$
V_{k} v \rightarrow V v \quad \text { in } L^{1}\left(\Omega ; d_{\partial \Omega} \mathrm{d} x\right) .
$$

The lemma then follows by letting $k \rightarrow \infty$ in (3.3).

Lemma 3.4. Assume that $V \in L^{q}(\Omega)$ for some $q>N$. Then the boundary value problem (1.7) has a distributional solution for every $\nu \in \mathcal{M}(\partial \Omega)$. In particular, $\Sigma=\emptyset$.

We recall that if $v \in L^{1}(\Omega), f \in L^{1}\left(\Omega ; d_{\partial \Omega} \mathrm{d} x\right)$ and $\nu \in \mathcal{M}(\partial \Omega)$ satisfy

$$
-\int_{\Omega} v \Delta \zeta \mathrm{d} x=\int_{\Omega} f \zeta \mathrm{d} x+\int_{\partial \Omega} \frac{\partial \zeta}{\partial n} \mathrm{~d} \nu \text { for every } \zeta \in C_{0}^{\infty}(\bar{\Omega}),
$$

which is the weak formulation of $v$ being a solution of

$$
\left\{\begin{aligned}
-\Delta v=f & \text { in } \Omega, \\
v=\nu & \text { on } \partial \Omega,
\end{aligned}\right.
$$

then

(i) for every $1 \leq p<\frac{N}{N-1}$, we have $v \in L^{p}(\Omega)$ and there exists a constant $C>0$ depending on $p$ and $\Omega$ such that

$$
\|v\|_{L^{p}(\Omega)} \leq C\left(\|f\|_{L^{1}\left(\Omega ; d_{\partial \Omega} \mathrm{d} x\right)}+\|\nu\|_{\mathcal{M}(\partial \Omega)}\right) ;
$$

(ii) for every $1 \leq p<\frac{N}{N-1}$ and every $\omega \Subset \Omega$, we have $\nabla v \in L^{p}\left(\omega ; \mathbb{R}^{N}\right)$ and there exists a constant $C^{\prime}>0$ depending on $p$ and $\omega$ such that

$$
\|\nabla v\|_{L^{p}\left(\omega ; \mathbb{R}^{N}\right)} \leq C^{\prime}\left(\|f\|_{L^{1}\left(\Omega ; d_{\partial \Omega} \mathrm{d} x\right)}+\|\nu\|_{\mathcal{M}(\partial \Omega)}\right) .
$$

We refer the reader to [15, Theorem 1.2.2] for a proof of these assertions.

Proof of Lemma 3.4. Let $\left(g_{k}\right)$ be a sequence of smooth functions on $\partial \Omega$ such that $\left\|g_{k}\right\|_{L^{1}(\partial \Omega)} \rightarrow\|\nu\|_{\mathcal{M}(\partial \Omega)}$ and $g_{k}{ }^{*} \nu$ weak ${ }^{*}$ in $\mathcal{M}(\partial \Omega)$, i.e.,

$$
\lim _{k \rightarrow \infty} \int_{\partial \Omega} \phi g_{k} \mathrm{~d} x=\int_{\partial \Omega} \phi \mathrm{d} \nu \text { for every } \phi \in C(\partial \Omega) .
$$

Such a sequence can be obtained, for example, from a convolution of $\nu$ with a sequence of mollifiers. Denote by $v_{k}$ the distributional solution of (1.7) associated to $g_{k}$. Given $\omega \Subset \Omega$, we deduce from (3.4) and (3.5) that

$$
\left\|v_{k}\right\|_{W^{1,1}(\omega)} \leq C_{1}\left(\left\|V v_{k}\right\|_{L^{1}\left(\Omega ; d_{\partial \Omega} \mathrm{d} x\right)}+\left\|g_{k}\right\|_{L^{1}(\partial \Omega)}\right)
$$


for some constant $C_{1}>0$ depending on $\omega$. Taking a subsequence if necessary, we have

$$
v_{k} \rightarrow v \quad \text { almost everywhere on } \Omega \text {. }
$$

On the other hand, since $q^{\prime}<\frac{N}{N-1}$, we deduce from (3.4) that

$$
\left\|v_{k}\right\|_{L^{q^{\prime}}(\Omega)} \leq C_{2}\left(\left\|V v_{k}\right\|_{L^{1}\left(\Omega ; d_{\partial \Omega} \mathrm{d} x\right)}+\left\|g_{k}\right\|_{L^{1}(\partial \Omega)}\right)
$$

for some constant $C_{2}>0$ depending on $q$ and $\Omega$. Hence $\left(v_{k}\right)$ is bounded in $L^{q^{\prime}}(\Omega)$. This, together with (3.6), implies that

$$
v_{k} \rightarrow v \text { weakly in } L^{q^{\prime}}(\Omega) .
$$

Recalling that $V \in L^{q}(\Omega)$ and taking the limit as $k \rightarrow \infty$ in the equation

$$
\int_{\Omega} v_{k}(-\Delta \zeta+V \zeta) \mathrm{d} x=\int_{\Omega} \frac{\partial \zeta}{\partial n} g_{k} \mathrm{~d} \sigma \quad \text { for every } \zeta \in C_{0}^{\infty}(\bar{\Omega}),
$$

we get the conclusion.

We now turn to the

Proof of Proposition 3.2. We first assume that $V$ is bounded. In this case, $\zeta_{f} \in C_{0}^{1}(\bar{\Omega})$ for every $f \in L^{\infty}(\Omega)$. Since $\zeta_{f}$ need not be smooth enough to be used as test function for $v$, we approximate $\zeta_{f}$ in $C^{1}(\bar{\Omega})$ by a sequence $\left(\zeta_{f_{k}}\right)$ in $C_{0}^{\infty}(\bar{\Omega})$, where $\left(f_{k}\right)$ is a bounded sequence in $L^{\infty}(\Omega)$ such that $f_{k} \rightarrow f$ almost everywhere on $\Omega$. For this purpose, we follow the construction given in [17]: for each $k$ we define the function $g_{k}=\rho_{k} * g$, where $g=f-V \zeta_{f}$ and $\left(\rho_{k}\right)$ is a sequence of mollifiers, and we denote by $w_{k} \in C_{0}^{\infty}(\bar{\Omega})$ the solution of

$$
\left\{\begin{aligned}
-\Delta w_{k}=g_{k} & \text { in } \Omega, \\
w_{k}=0 & \text { on } \partial \Omega .
\end{aligned}\right.
$$

Observe that $w_{k}=\zeta_{f_{k}}$ with $f_{k}=g_{k}+V w_{k}$. Moreover, estimate (2.4) ensures that for some fixed $N<p<\infty$,

$$
\left\|\zeta_{f_{k}}-\zeta_{f}\right\|_{C^{1}(\bar{\Omega})} \leq C\left\|g_{k}-g\right\|_{L^{p}(\Omega)} .
$$

Letting $k \rightarrow \infty$ in this estimate, we have

$$
\zeta_{f_{k}} \rightarrow \zeta_{f} \quad \text { uniformly on } \Omega \text { and } \quad \frac{\partial \zeta_{f_{k}}}{\partial n} \rightarrow \frac{\partial \zeta_{f}}{\partial n} \quad \text { uniformly on } \partial \Omega \text {. }
$$

Since

$$
\int_{\Omega} v f_{k} \mathrm{~d} x=\int_{\Omega} v\left(-\Delta \zeta_{f_{k}}+V \zeta_{f_{k}}\right) \mathrm{d} x=\int_{\partial \Omega} \frac{\partial \zeta_{f_{k}}}{\partial n} \mathrm{~d} \nu
$$

taking the limit as $k \rightarrow \infty$, we obtain

$$
\int_{\Omega} v f \mathrm{~d} x=\int_{\partial \Omega} \frac{\partial \zeta_{f}}{\partial n} \mathrm{~d} \nu
$$

In the general case where $V \in L_{\text {loc }}^{1}(\Omega)$, let $v_{k}$ be the solution of

$$
\left\{\begin{aligned}
-\Delta v_{k}+V_{k} v_{k}=0 & \text { in } \Omega, \\
v_{k}=\nu & \text { on } \partial \Omega,
\end{aligned}\right.
$$


whose existence is ensured by Lemma 3.4. Given $f \in L^{\infty}(\Omega)$, we denote by $z_{k} \in C_{0}^{1}(\bar{\Omega})$ the solution of

$$
\left\{\begin{aligned}
-\Delta z_{k}+V_{k} z_{k}=f & \text { in } \Omega, \\
z_{k}=0 & \text { on } \partial \Omega .
\end{aligned}\right.
$$

It follows from the first part of the proof that

$$
\int_{\Omega} v_{k} f \mathrm{~d} x=\int_{\partial \Omega} \frac{\partial z_{k}}{\partial n} \mathrm{~d} \nu
$$

Proposition 2.1 implies that $\left(\partial z_{k} / \partial n\right)$ is uniformly bounded and converges pointwise to $\widehat{\partial \zeta_{f}} / \partial n$ on $\partial \Omega$. By Lemma 3.3, we obtain

$$
\int_{\Omega} v f \mathrm{~d} x=\int_{\partial \Omega} \frac{\widehat{\partial \zeta_{f}}}{\partial n} \mathrm{~d} \nu
$$

\section{Pointwise Normal DeRIVATIVE ON THE EXCEPtional SET $\Sigma$}

In this section, we characterize the exceptional boundary set $\Sigma$ using the pointwise normal derivative with respect to the Schrödinger operator $-\Delta+V$ introduced in Section 2. We prove that

Proposition 4.1. For every $a \in \partial \Omega$, we have $a \in \Sigma$ if and only if

$$
\frac{\widehat{\partial \zeta_{f}}}{\partial n}(a)=0 \quad \text { for every } f \in L^{\infty}(\Omega) .
$$

As a fundamental property that is used in the proofs of Proposition 4.1 and Theorem 3, we first extend Lemma 3.3 to the case where (1.7) need not have a distributional solution.

Proposition 4.2. Let $\nu \in \mathcal{M}(\partial \Omega)$ be a nonnegative measure and let $v$ be the duality solution of (1.7) associated to $\nu$. We have that

(i) if $v_{k}$ is the distributional solution of (3.1), then $v_{k} \rightarrow v$ in $L^{1}(\Omega)$;

(ii) there exists a nonnegative measure $\lambda \in \mathcal{M}(\partial \Omega)$ such that $v$ is the distributional solution of (1.7) associated to $\nu-\lambda$.

We recall the following estimate whose proof is sketched for the convenience of the reader:

Lemma 4.3. If $v$ is a distributional solution of (1.7) with $\nu \in \mathcal{M}(\partial \Omega)$, then

$$
\|v\|_{L^{1}(\Omega)}+\|V v\|_{L^{1}\left(\Omega ; d_{\partial \Omega} \mathrm{d} x\right)} \leq C\|\nu\|_{\mathcal{M}(\partial \Omega)}
$$

for some constant $C>0$ depending on $\Omega$.

Proof of Lemma 4.3. One deduces using Kato's inequality (2.10) with $w=v$ and $w=-v$ that

$$
\int_{\Omega}|v|(-\Delta \zeta+V \zeta) \mathrm{d} x \leq\left\|\frac{\partial \zeta}{\partial n}\right\|_{L^{\infty}(\partial \Omega)}\|\nu\|_{\mathcal{M}(\partial \Omega)}
$$


for every $\zeta \in C_{0}^{\infty}(\bar{\Omega}), \zeta \geq 0$ on $\bar{\Omega}$. Take as test function the solution $\theta$ of (3.2). As a consequence of the classical Hopf lemma, there exists $C_{1}>0$ such that $\theta \geq C_{1} d_{\partial \Omega}$ on $\Omega$. Therefore, by nonnegativity of $V$,

$$
\|v\|_{L^{1}(\Omega)}+C_{1}\|V v\|_{L^{1}\left(\Omega ; d_{\partial \Omega} \mathrm{d} x\right)} \leq\left\|\frac{\partial \theta}{\partial n}\right\|_{L^{\infty}(\partial \Omega)}\|\nu\|_{\mathcal{M}(\partial \Omega)} .
$$

Proof of Proposition 4.2. By a straightforward counterpart of Lemmas 2.2 and 2.3 for distributional solutions of (1.7), the sequence $\left(v_{k}\right)$ is nonnegative and non-increasing. Hence $\left(v_{k}\right)$ converges in $L^{1}(\Omega)$ to some nonnegative function $w$. Let $f \in L^{\infty}(\Omega)$ and let $u_{k}$ be the solution of (2.3). Proposition 3.2 implies that

$$
\int_{\Omega} v_{k} f \mathrm{~d} x=\int_{\partial \Omega} \frac{\partial u_{k}}{\partial n} \mathrm{~d} \nu
$$

By Proposition 2.1, the sequence $\left(\partial u_{k} / \partial n\right)$ is uniformly bounded and converges pointwise to $\widehat{\partial \zeta_{f}} / \partial n$ on $\partial \Omega$. Taking the limit as $k \rightarrow \infty$ in the identity above, we deduce from Lebesgue's dominated convergence theorem that

$$
\int_{\Omega} w f \mathrm{~d} x=\int_{\partial \Omega} \frac{\widehat{\partial \zeta_{f}}}{\partial n} \mathrm{~d} \nu \text { for every } f \in L^{\infty}(\Omega) .
$$

We have thus proved that $w$ is a duality solution of (1.7) involving $\nu$. By uniqueness of duality solutions, we have $v=w$.

For every $k \geq 1$, we have

$$
0 \leq V_{k} v_{k} \leq V v_{1} \text { almost everywhere on } \Omega \text {. }
$$

Since $v_{1}$ is subharmonic, it is locally bounded on $\Omega$; see [21, Theorem 8.1.5]. Then, Lebesgue's dominated convergence theorem implies that

$$
V_{k} v_{k} \rightarrow V v \quad \text { in } L_{\mathrm{loc}}^{1}(\Omega) .
$$

Let $\theta$ be the unique solution of (3.2). Since $0<\theta \leq\|\nabla \theta\|_{L^{\infty}(\Omega)} d_{\partial \Omega}$ on $\Omega$, by Lemma 4.3 the sequence $\left(V_{k} v_{k} \theta\right)$ is bounded in $L^{1}(\Omega)$. Therefore, taking a subsequence if necessary, we may assume that there exist nonnegative finite Borel measures $\mu$ on $\Omega$ and $\tau$ on $\partial \Omega$ such that, for every $\psi \in C^{\infty}(\bar{\Omega})$,

$$
\lim _{k \rightarrow \infty} \int_{\Omega} V_{k} v_{k} \theta \psi \mathrm{d} x=\int_{\Omega} \psi \mathrm{d} \mu+\int_{\partial \Omega} \psi \mathrm{d} \tau .
$$

On the other hand, for every $\varphi \in C_{c}^{\infty}(\Omega)$,

$$
\lim _{k \rightarrow \infty} \int_{\Omega} V_{k} v_{k} \theta \varphi \mathrm{d} x=\int_{\Omega} V v \theta \varphi \mathrm{d} x .
$$

Hence $\mu=V v \theta \mathrm{d} x$. Given $\zeta \in C_{0}^{\infty}(\bar{\Omega})$, we define $\gamma=\zeta / \theta$ on $\Omega$. Since $\partial \theta / \partial n>0$ on $\partial \Omega$, the function $\gamma$ extends continuously to $\partial \Omega$, and

$$
\gamma=\frac{\partial \zeta}{\partial n} \frac{1}{\frac{\partial \theta}{\partial n}} \quad \text { on } \partial \Omega
$$


Taking $\psi=\gamma$ in (4.1), we obtain

$$
\lim _{k \rightarrow \infty} \int_{\Omega} V_{k} v_{k} \zeta \mathrm{d} x=\int_{\Omega} V v \zeta \mathrm{d} x+\int_{\partial \Omega} \frac{\partial \zeta}{\partial n} \frac{1}{\frac{\partial \theta}{\partial n}} \mathrm{~d} \tau \quad \text { for every } \zeta \in C_{0}^{\infty}(\bar{\Omega}) .
$$

The result follows with $\lambda=\frac{1}{\frac{\partial \theta}{\partial n}} \tau$.

Another ingredient involved in the proof of Proposition 4.1 is the inverse maximum principle for distributional solutions of (1.7); see [6, Lemma 1].

Lemma 4.4. Let $\nu \in \mathcal{M}(\partial \Omega)$ and let $h \in L^{1}\left(\Omega ; d_{\partial \Omega} \mathrm{d} x\right)$. Assume that $v \in L^{1}(\Omega)$ satisfies

$$
-\int_{\Omega} v \Delta \zeta \mathrm{d} x=\int_{\Omega} h \zeta \mathrm{d} x+\int_{\partial \Omega} \frac{\partial \zeta}{\partial n} \mathrm{~d} \nu \quad \text { for every } \zeta \in C_{0}^{\infty}(\bar{\Omega}) .
$$

If $v \geq 0$ almost everywhere on $\Omega$, then $\nu \geq 0$ on $\partial \Omega$.

Given $a \in \partial \Omega$, we denote by $P_{a}$ the duality solution of (1.7) associated to the Dirac measure $\delta_{a}$, that is,

$$
\frac{\widehat{\partial \zeta_{f}}}{\partial n}(a)=\int_{\Omega} P_{a} f \mathrm{~d} x \quad \text { for every } f \in L^{\infty}(\Omega) .
$$

One deduces from the definition of duality solution using $f=\chi_{\left\{P_{a}<0\right\}}$ as test function that

$$
P_{a} \geq 0 \quad \text { almost everywhere on } \Omega \text {. }
$$

We apply this simple observation in the

Proof of Proposition 4.1. We first assume that $a \in \Sigma$. By Proposition 4.2, $P_{a}$ is a distributional solution of (1.7) with datum $\delta_{a}-\lambda$ for some nonnegative measure $\lambda \in \mathcal{M}(\partial \Omega)$. Since $P_{a} \geq 0$ almost everywhere on $\Omega$, we deduce from Lemma 4.4 that

$$
\delta_{a} \geq \lambda \geq 0 \quad \text { on } \partial \Omega \text {. }
$$

Thus, $\lambda=\alpha \delta_{a}$ for some $0 \leq \alpha \leq 1$. If we had $\alpha \neq 1$, then $P_{a} /(1-\alpha)$ would be a distributional solution of (1.4), in contradiction with the assumption that $a \in \Sigma$. Hence, $\alpha=1$ and

$$
\int_{\Omega} P_{a}(-\Delta \zeta+V \zeta) \mathrm{d} x=0 \quad \text { for every } \zeta \in C_{0}^{\infty}(\bar{\Omega}) .
$$

Taking $\zeta=\theta$, where $\theta$ satisfies (3.2), we deduce that

$$
\int_{\Omega} P_{a} \mathrm{~d} x=0 .
$$

Since $P_{a}$ is nonnegative, we have $P_{a}=0$ almost everywhere on $\Omega$. The representation formula (4.2) then implies that

$$
\frac{\widehat{\partial \zeta_{f}}}{\partial n}(a)=0 \quad \text { for every } f \in L^{\infty}(\Omega) .
$$


For the converse, one deduces from the assumption on $a$ and (4.2) applied to $f \equiv 1$ that

$$
\int_{\Omega} P_{a} \mathrm{~d} x=0 .
$$

Hence $P_{a}=0$ almost everywhere on $\Omega$, so that $P_{a}$ cannot be a distributional solution of (1.7) involving $\delta_{a}$. Since $P_{a}$ is the only candidate for such a solution due to Proposition 3.2, we conclude that $a \in \Sigma$.

\section{Proof of Theorem 3}

$(\Leftarrow)$. Let $v$ be the duality solution of (1.7) associated to $\nu$. Proposition 4.2 implies the existence of a nonnegative measure $\lambda \in \mathcal{M}(\partial \Omega)$ such that $v$ is a distributional solution of (1.7) involving $\nu-\lambda$. We claim that $\lambda(\partial \Omega \backslash \Sigma)=0$. By Proposition 3.2, $v$ is also a duality solution of (1.7) with datum $\nu-\lambda$. Hence

$$
\int_{\partial \Omega} \frac{\widehat{\partial \zeta_{f}}}{\partial n} \mathrm{~d} \nu=\int_{\Omega} v f \mathrm{~d} x=\int_{\partial \Omega} \frac{\widehat{\partial \zeta_{f}}}{\partial n} \mathrm{~d}(\nu-\lambda) \quad \text { for every } f \in L^{\infty}(\Omega),
$$

which implies that

$$
\int_{\partial \Omega} \frac{\widehat{\partial \zeta_{f}}}{\partial n} \mathrm{~d} \lambda=0 \quad \text { for every } f \in L^{\infty}(\Omega)
$$

By Proposition 4.1, we have $\widehat{\partial \zeta_{1}} / \partial n>0$ on $\partial \Omega \backslash \Sigma$. Since $\lambda$ is nonnegative, we conclude that $\lambda(\partial \Omega \backslash \Sigma)=0$ as claimed. On the other hand, since $v \geq 0$ almost everywhere on $\Omega$, we deduce from Lemma 4.4 that

$$
\nu \geq \lambda \geq 0 \quad \text { on } \partial \Omega .
$$

By assumption, $\nu(\Sigma)=0$. Hence $\lambda(\Sigma)=0$. We thus have

$$
\lambda(\partial \Omega)=\lambda(\partial \Omega \backslash \Sigma)+\lambda(\Sigma)=0,
$$

that is, $\lambda=0$.

$(\Rightarrow)$. Let $v$ be the distributional solution of (1.7) associated to $\nu$. Proposition 3.2 implies that $v$ is also a duality solution of (1.7) involving the same datum. By Proposition 4.1, we have

$$
\int_{\Omega} v f \mathrm{~d} x=\int_{\partial \Omega} \frac{\widehat{\partial \zeta_{f}}}{\partial n} \mathrm{~d} \nu=\int_{\partial \Omega} \frac{\widehat{\partial \zeta_{f}}}{\partial n} \mathrm{~d} \nu L_{\partial \Omega \backslash \Sigma} \quad \text { for every } f \in L^{\infty}(\Omega),
$$

so that $v$ is also a duality solution of (1.7) with datum $\nu\left\lfloor_{\partial \Omega \backslash \Sigma}\right.$. The reverse implication in Theorem 3 implies that (1.7) associated to $\nu\left\lfloor_{\partial \Omega \backslash \Sigma}\right.$ has a unique distributional solution $z$. But then, Proposition 3.2 ensures that $z$ is also a duality solution of (1.7) with datum $\nu\left\lfloor_{\partial \Omega \backslash \Sigma}\right.$. Since duality solutions are unique, we have $v=z$ almost everywhere on $\Omega$. Thus, $v$ is a distributional solution of (1.7) with both $\nu$ and $\nu\left\lfloor_{\partial \Omega \backslash \Sigma}\right.$, which implies that $\nu=\nu\left\lfloor_{\partial \Omega \backslash \Sigma}\right.$, and then $\nu(\Sigma)=0$. 
Remark 5.1. Using Theorem 3 and Proposition 4.1, we can now explain why $\widehat{\partial u} / \partial n$ does not depend upon the particular choice of approximating sequence $\left(V_{k}\right)$ in Proposition 2.1. Indeed, we have by Proposition 4.1 that

$$
\widehat{\partial u}(a)=0 \quad \text { for every } a \in \Sigma,
$$

while $\Sigma$ is independent of $\left(V_{k}\right)$. When $a \in \partial \Omega \backslash \Sigma$, it follows from Theorem 3 that $P_{a}$ is a distributional solution of (1.4), whose definition does not involve $\left(V_{k}\right)$. Thus, by the representation formula $(4.2), \widehat{\partial u} / \partial n$ is independent of $\left(V_{k}\right)$ also on $\partial \Omega \backslash \Sigma$.

\section{Proof of Theorem 1}

We deduce Theorem 1 as a consequence of

Proposition 6.1. Let $u$ be the solution of (1.1) for some nonnegative datum in $L^{\infty}(\Omega)$ such that $\hat{u}$ has a classical normal derivative at $a \in \partial \Omega$ which satisfies (1.3). If $v$ is another solution of (1.1) for some nonnegative datum in $L^{\infty}(\Omega)$, and if $v \leq u$ almost everywhere on $\Omega$, then $\hat{v}$ also has a classical normal derivative at a which satisfies (1.3).

We recall that whenever $v$ satisfies (1.1) with datum $h \in L^{\infty}(\Omega)$, one has

$$
\hat{v}(x)=\int_{\Omega} G(x, y)(h-V v)(x) \mathrm{d} x \quad \text { for every } x \in \Omega .
$$

Using standard estimates on the Green function $G$, by boundedness of $h$ one shows that

$$
\lim _{t \downarrow 0} \int_{\Omega} \frac{G(a+t n, y)}{t} h(y) \mathrm{d} y=\int_{\Omega} K(a, y) h(y) \mathrm{d} y,
$$

where $n=n(a)$. The main difficulty in the proof of Proposition 6.1 thus consists in getting that

$$
\lim _{t \downarrow 0} \int_{\Omega} \frac{G(a+t n, y)}{t} V v(y) \mathrm{d} y=\int_{\Omega} K(a, y) V v(y) \mathrm{d} y .
$$

Proof of Proposition 6.1. Let $\left(\varepsilon_{k}\right)$ be a non-increasing sequence of positive numbers converging to 0 . We define on $\Omega$

$$
g_{k}(y)=\frac{G\left(a+\varepsilon_{k} n, y\right)}{\varepsilon_{k}} \text {. }
$$

On the one hand, we have $g_{k}(y) \rightarrow K(a, y)$ for all $y \in \Omega$. On the other hand, by assumption on $u$,

$$
\int_{\Omega} g_{k}(y) V u(y) \mathrm{d} y \rightarrow \int_{\Omega} K(a, y) V u(y) \mathrm{d} y
$$

or, equivalently,

$$
\left\|g_{k} u\right\|_{L^{1}(\Omega ; V \mathrm{~d} x)} \rightarrow\|K(a, .) u\|_{L^{1}(\Omega ; V \mathrm{~d} x)} .
$$


We thus have pointwise convergence of $\left(g_{k} u\right)$ and also convergence of norms. Therefore,

$$
g_{k} u \rightarrow K(a, .) u \quad \text { in } L^{1}(\Omega ; V \mathrm{~d} x),
$$

which is a special case of the Brezis-Lieb lemma [3]; see [21, Proposition 4.2.6]. Since $0 \leq v \leq u$, we deduce from Lebesgue's dominated convergence theorem that $g_{k} v \rightarrow K(a,)$.$v in L^{1}(\Omega ; V \mathrm{~d} x)$. The conclusion is now straightforward.

For the proof of Theorem 1, we also need the following

Lemma 6.2. Let $u$ be the solution of (1.1) for some nonnegative datum $f \in L^{\infty}(\Omega)$. Then, for every $a \in \partial \Omega$, we have

$$
\limsup _{\varepsilon \downarrow 0} \frac{\hat{u}(a+\varepsilon n)}{\varepsilon} \leq \frac{\widehat{\partial u}}{\partial n}(a) \leq \int_{\Omega} K(a, y)(f-V u)(y) \mathrm{d} y .
$$

Proof of Lemma 6.2. Let $u_{k}$ satisfy (2.3). By comparison, we have $\hat{u} \leq u_{k}$ on $\Omega$. Hence,

$$
\limsup _{\varepsilon \downarrow 0} \frac{\hat{u}(a+\varepsilon n)}{\varepsilon} \leq \frac{\partial u_{k}}{\partial n}(a) \quad \text { for every } a \in \partial \Omega .
$$

Then, as $k \rightarrow \infty$ we deduce the first inequality in the statement.

Next, since $\Delta u_{k}$ is bounded, for every $a \in \partial \Omega$ we have

$$
\frac{\partial u_{k}}{\partial n}(a)=\int_{\Omega} K(a, y)\left(-\Delta u_{k}(y)\right) \mathrm{d} y=\int_{\Omega} K(a, y)\left(f-V_{k} u_{k}\right)(y) \mathrm{d} y .
$$

By Proposition 2.1, the sequence $\left(V_{k} u_{k}\right)$ converges almost everywhere to $V u$. Fatou's lemma implies that, for every $a \in \partial \Omega$,

$$
\int_{\Omega} K(a, y) V u(y) \mathrm{d} y \leq \liminf _{k \rightarrow \infty} \int_{\Omega} K(a, y) V_{k} u_{k}(y) \mathrm{d} y .
$$

Observe that the right-hand side is finite since the sequence $\left(\partial u_{k} / \partial n\right)$ is bounded. Hence, for every $a \in \partial \Omega$, we have

$$
\begin{aligned}
\frac{\widehat{\partial u}}{\partial n}(a)=\lim _{k \rightarrow \infty} \frac{\partial u_{k}}{\partial n}(a) & =\lim _{k \rightarrow \infty} \int_{\Omega} K(a, y)\left(f-V_{k} u_{k}\right)(y) \mathrm{d} y \\
& \leq \int_{\Omega} K(a, y)(f-V u)(y) \mathrm{d} y,
\end{aligned}
$$

which implies the second inequality in the statement.

Proof of Theorem 1. The reverse implication $(\Leftarrow)$ follows from Proposition 6.1 and the fact that $u \leq \zeta_{\|f\|_{L^{\infty}(\Omega)}}=\|f\|_{L^{\infty}(\Omega)} \zeta_{1}$ almost everywhere on $\Omega$. We now prove the direct implication $(\Rightarrow)$. One shows the existence of a constant $C>0$ such that, for every $\varepsilon>0$, the solution $v_{\varepsilon}$ of the Dirichlet problem

$$
\left\{\begin{aligned}
-\Delta v_{\varepsilon}+V v_{\varepsilon} & \left.=\chi_{\{u / C>\varepsilon\}}\right\} & & \text { in } \Omega, \\
v_{\varepsilon} & =0 & & \text { on } \partial \Omega,
\end{aligned}\right.
$$


satisfies $v_{\varepsilon} \leq u / \varepsilon$ almost everywhere on $\Omega$; this is a consequence of Kato's inequality, as explained in the proof of Proposition 4.1 in [16]. Let $\left(\varepsilon_{j}\right)$ be a non-increasing sequence of positive numbers converging to 0 . Since $\chi_{\left\{u / C>\varepsilon_{j}\right\}} \leq 1$, by Proposition 6.1 the function $\widehat{v_{\varepsilon_{j}}}$ has a normal derivative at $a$ which satisfies (1.3), which means that

$$
\frac{\partial \widehat{\varepsilon_{\varepsilon_{j}}}}{\partial n}(a)=\int_{\Omega} K(a, y)\left(\chi_{\left\{u / C>\varepsilon_{j}\right\}}-V v_{\varepsilon_{j}}\right)(y) \mathrm{d} y .
$$

By the strong maximum principle for the SchrÂúdinger operator with potential in $L_{\text {loc }}^{1}(\Omega)$, we have $u>0$ almost everywhere on $\Omega$. Hence

$$
\chi_{\left\{u / C>\varepsilon_{j}\right\}} \rightarrow 1 \text { almost everywhere on } \Omega .
$$

The convergence thus holds in $L^{1}(\Omega)$, which implies that

$$
v_{\varepsilon_{j}} \rightarrow \zeta_{1} \quad \text { in } L^{1}(\Omega)
$$

As the sequence $\left(v_{\varepsilon_{j}}\right)$ is nondecreasing, we deduce from Levi's monotone convergence theorem that

$$
\lim _{j \rightarrow \infty} \frac{\partial \widehat{v_{\varepsilon_{j}}}}{\partial n}(a)=\int_{\Omega} K(a, y)\left(1-V \zeta_{1}(y)\right) \mathrm{d} y .
$$

Since $\widehat{v_{\varepsilon_{j}}} \leq \widehat{\zeta_{1}}$ on $\Omega$, we also have, by classical comparison of limits,

$$
\lim _{j \rightarrow \infty} \frac{\partial \widehat{\varepsilon_{\varepsilon_{j}}}}{\partial n}(a) \leq \liminf _{j \rightarrow \infty} \frac{\widehat{\zeta_{1}}\left(a+\varepsilon_{j} n\right)}{\varepsilon_{j}} .
$$

Lemma 6.2 implies that

$$
\liminf _{k \rightarrow \infty} \frac{\widehat{\zeta_{1}}\left(a+\varepsilon_{k} n\right)}{\varepsilon_{k}} \leq \limsup _{k \rightarrow \infty} \frac{\widehat{\zeta}_{1}\left(a+\varepsilon_{k} n\right)}{\varepsilon_{k}} \leq \int_{\Omega} K(a, y)\left(1-V \zeta_{1}(y)\right) \mathrm{d} y .
$$

Combining the inequalities above, we deduce that $\partial \widehat{\zeta}_{1}(a) / \partial n$ exists and

$$
\frac{\partial \widehat{\zeta_{1}}}{\partial n}(a)=\lim _{k \rightarrow \infty} \frac{\partial \widehat{\varepsilon_{k}}}{\partial n}(a)=\int_{\Omega} K(a, y)\left(1-V \zeta_{1}(y)\right) \mathrm{d} y .
$$

Hence, by definition, $a \in \mathcal{N}$.

\section{Proof of THEOREM 2}

We first prove a version of the Hopf lemma in terms of the pointwise normal derivative associated to the Schrödinger operator $-\Delta+V$. In this case, the answer does not involve the set $\mathcal{N}$.

Proposition 7.1. Let $u$ be the solution of (1.1) for some nonnegative datum $f \in L^{\infty}(\Omega), f \not \equiv 0$. Then, for every $a \in \partial \Omega$, we have

$$
\frac{\partial u}{\partial n}(a)>0 \quad \text { if and only if } a \notin \Sigma \text {. }
$$


Proof. By Proposition 4.1, we only have to prove that $\widehat{\partial u} / \partial n>0$ on $\partial \Omega \backslash \Sigma$. For this purpose, let $a \in \partial \Omega \backslash \Sigma$. In this case, $P_{a}$ is both a duality and a distributional solution of (1.7) involving $\delta_{a}$. As a distributional solution, it satisfies the strong maximum principle for the Schrödinger operator with potential in $L_{\text {loc }}^{1}(\Omega)$. Hence,

$$
P_{a}>0 \text { almost everywhere on } \Omega \text {. }
$$

As a duality solution, $P_{a}$ satisfies the representation formula (4.2). Since $f$ is nonzero, we then deduce from this formula and (7.1) that

$$
\widehat{\partial u}(a)>0 \text {. }
$$

We now turn to the

Proof of Theorem 2. By Theorem 1, the classical normal derivative $\partial \hat{u} / \partial n$ exists on $\mathcal{N}$ and satisfies (1.3). A direct application of Lemma 6.2 gives, for every $a \in \mathcal{N}$,

$$
\frac{\partial \hat{u}}{\partial n}(a) \leq \frac{\widehat{\partial u}}{\partial n}(a) \leq \int_{\Omega} K(a, y)(f-V u)(y) \mathrm{d} y .
$$

As the integral in the right-hand side equals $\partial \hat{u}(a) / \partial n$, equality holds everywhere and we get

$$
\frac{\partial \hat{u}}{\partial n}=\frac{\widehat{\partial u}}{\partial n} \quad \text { on } \mathcal{N}
$$

The theorem then follows from Proposition 7.1.

We conclude this section with the following particular case of Theorem 2 .

Corollary 7.2. Assume that $V \in L^{q}(\Omega)$ for some $q>N$. Then, for every solution $u$ of (1.1) involving a nonnegative datum $f \in L^{\infty}(\Omega), f \not \equiv 0$, the normal derivative of $\hat{u}$ exists at every point $a \in \partial \Omega$ and satisfies

$$
\frac{\partial \hat{u}}{\partial n}(a)>0 .
$$

Proof. We prove that $\mathcal{N}=\partial \Omega$ and $\Sigma=\emptyset$. Let $u$ be the solution of (1.1) involving some nonnegative datum $f \in L^{\infty}(\Omega)$. Since $u \in L^{\infty}(\Omega)$, we have $\Delta u \in L^{q}(\Omega)$, and then $u \in W^{2, q}(\Omega)$. The Morrey-Sobolev embedding theorem ensures that $u \in C^{1}(\bar{\Omega})$, which gives $\mathcal{N}=\partial \Omega$. That $\Sigma=\emptyset$ follows from Lemma 3.4. We then have the conclusion using Theorem 2 .

\section{ACKNOWLEDGEMENTS}

The first author (A.C.P.) was supported by the Fonds de la Recherche scientifique (F.R.S.-FNRS) under research grant J.0020.18. 


\section{REFERENCES}

[1] Alano Ancona, Une propriété d'invariance des ensembles absorbants par perturbation d'un opérateur elliptique, Comm. Partial Differential Equations 4 (1979), no. 4, 321337. $\uparrow 3$

[2] _ Positive solutions of Schrödinger equations and fine regularity of boundary points, Math. Z. 272 (2012), no. 1-2, 405-427. $\uparrow 3$

[3] Haïm Brezis and Elliott Lieb, A relation between pointwise convergence of functions and convergence of functionals, Proc. Amer. Math. Soc. 88 (1983), no. 3, 486-490. $\uparrow 18$

[4] Haïm Brezis, Moshe Marcus, and Augusto C. Ponce, Nonlinear elliptic equations with measures revisited, Mathematical aspects of nonlinear dispersive equations, Ann. of Math. Stud., vol. 163, Princeton Univ. Press, Princeton, NJ, 2007, pp. 55-109. $\uparrow 7$

[5] Haïm Brezis and Augusto C. Ponce, Remarks on the strong maximum principle, Differential Integral Equations 16 (2003), no. 1, 1-12. $\uparrow 3$

[6] _ Reduced measures on the boundary, J. Funct. Anal. 229 (2005), no. 1, 95-120. $\uparrow 15$

[7] _ Kato's inequality up to the boundary, Commun. Contemp. Math. 10 (2008), no. $6,1217-1241 . \uparrow 5$

[8] Jesús Ildefonso Díaz, On the ambiguous treatment of the Schrödinger equation for the infinite potential well and an alternative via singular potentials: the multi-dimensional case, SeMA J. 74 (2017), no. 3, 255-278. Erratum: SeMA J. 75, no. 3, 563-568. $\uparrow 4$

[9] Jesús Ildefonso Díaz and Jean-Michel Rakotoson, On the differentiability of very weak solutions with right-hand side data integrable with respect to the distance to the boundary, J. Funct. Anal. 257 (2009), no. 3, 807-831. $\uparrow 7$

[10] Lawrence C. Evans, Partial differential equations, 2nd ed., Graduate Studies in Mathematics, vol. 19, American Mathematical Society, Providence, RI, 2010. $\uparrow 1$

[11] David Gilbarg and Neil S. Trudinger, Elliptic partial differential equations of second order, Classics in Mathematics, Springer-Verlag, Berlin, 2001. $\uparrow 1,6$

[12] Walter Littman, Guido Stampacchia, and Hans F. Weinberger, Regular points for elliptic equations with discontinuous coefficients, Ann. Scuola Norm. Sup. Pisa (3) 17 (1963), 43-77. $\uparrow 7,9$

[13] Annalisa Malusa and Luigi Orsina, Existence and regularity results for relaxed Dirichlet problems with measure data, Ann. Mat. Pura Appl. (4) 170 (1996), 57-87. $\uparrow 4,9$

[14] Moshe Marcus and Laurent Véron, The boundary trace of positive solutions of semilinear elliptic equations: the supercritical case, J. Math. Pures Appl. (9) 77 (1998), no. 5, 481-524. $\uparrow 7$

[15] — Nonlinear second order elliptic equations involving measures, De Gruyter Series in Nonlinear Analysis and Applications, vol. 21, De Gruyter, Berlin, 2014. $\uparrow 7$, 11

[16] Luigi Orsina and Augusto C. Ponce, Hopf potentials for the Schrödinger operator, Anal. PDE 11 (2018), no. 8, 2015-2047. 119

[17] - On the nonexistence of Green's function and failure of the strong maximum principle. To appear in J. Math. Pures Appl. $\uparrow 4,12$

[18] Augusto C. Ponce, Elliptic PDEs, measures and capacities. From the Poisson equations to nonlinear Thomas-Fermi problems, EMS Tracts in Mathematics, vol. 23, European Mathematical Society (EMS), Zürich, 2016. $\uparrow 5$, 7, 9

[19] Neil S. Trudinger, On the positivity of weak supersolutions of nonuniformly elliptic equations, Bull. Austral. Math. Soc. 19 (1978), no. 3, 321-324. $\uparrow 3$ 
[20] Laurent Véron and Cecilia Yarur, Boundary value problems with measures for elliptic equations with singular potentials, J. Funct. Anal. 262 (2012), no. 3, 733-772. ^3, 4, 9

[21] Michel Willem, Functional analysis. Fundamentals and applications, Cornerstones, Birkhäuser/Springer, New York, 2013. ^6, 14, 18

Augusto C. Ponce

Université CATHolique de Louvain

Institut de Recherche en Mathématique et Physique

Chemin du Cyclotron 2, Bte L7.01.02

1348 Louvain-LA-Neuve

BELGIUM

E-mail address: Augusto.Ponce@uclouvain.be

Nicolas Wilmet

Université CATHOLIQUe de Louvain

Institut de Recherche en Mathématique et Physique

Chemin du Cyclotron 2, Bte L7.01.02

1348 Louvain-LA-Neuve

BELGIUM

E-mail address: Nicolas.Wilmet@uclouvain.be 